\title{
Teilen und Herrschen
}

Wettbewerb muss sein. Das gilt auch für die Arzneitherapie. Der Bundestagsausschuss für Jugend, Familie und Gesundheit formulierte die Notwendigkeit bei der Vorbereitung des "Gesetzes zur Neuordnung des Arzneimittelrechts" (2. Arzneimittelgesetz) wie folgt: "Nach einmütiger Auffassung des Ausschusses kann und darf es nicht Aufgabe des Gesetzgebers sein, durch die einseitige Festlegung bestimmter Methoden für den Nachweis der Wirksamkeit eines Arzneimittels eine der miteinander konkurrierenden Therapierichtungen ... zum ausschließlichen Maßstab... zu erheben." Das war am 24. August 1976 und ist nachzulesen in der Bundestagsdrucksache 7/5091/15.

Rückblickend wäre zu fragen: Hat die seinerzeit vom Parlament eingeforderte Konkurrenz der Therapierichtungen tatsächlich dazu beigetragen, dass Wirksamkeiten verbessert, Risiken minimiert oder Arzneikosten eingedämmt wurden? Bei genauerem Hinsehen durchaus! Erfolgreicher Wettbewerb zwischen zwei Therapierichtungen fand zum Beispiel auf dem Milliarden-Markt der Antidepressiva statt. Diese Gruppe von Wirkstoffen war seit 1957 ungestört und ertragssicher im Griff "schulmedizinischer" Synthetika. Anfang der Neunzigerjahre tauchte jedoch ein pflanzlicher Konkurrent auf. JohanniskrautExtrakte setzten neue Maßstäbe nicht nur bei der Verträglichkeit, sondern auch bei den Kosten. Mit mehr als 40 kontrollierten Therapiestudien wurde nachgewiesen, dass sich mit Extrakten aus Hypericum perforatum in der Praxis durchaus ähnliche Behandlungserfolge erzielen lassen wie mit herkömmlichen synthetischen Antidepressiva.

Betroffene Fachkreise reagierten geteilt. Die einen klammerten sich an die bekannte Maxime, dass nicht sein kann, was nicht sein darf. Bei anderen fand jedoch ein bemerkenswerter Wandel der Ansichten zur Arzneitherapie der Depressionen statt. Bis spät in die Neunzigerjahre wurde zum Beispiel in dem Periodikum Arzneiverordnungs-Report überwiegend löblich über neu in den Markt drängende synthetische Antidepressiva berichtet. Noch in der Ausgabe 2000 wurde wohlwollend von ninnovativen Arzneimittelgruppen wie ... selektiven Antidepressiva" berichtet (1). Danach änderte sich der Tenor. In der Ausgabe 2002 wurde erstmals eingeräumt: »Im Durchschnitt beträgt der absolute Unterschied der Responserate zwischen Antidepressiva und Placebo 20\%.... Breite Fortschritte in der Behandlung depressiver Patienten sind daher in den kommenden Jahren nicht von neuen Substanzen zu erwarten, sondern von ... rationalen Kombinations- bzw. Augmentationsstrategien« (2).
Etwa 50-70\% aller Patienten mit Depressionen sprechen erfahrungsgemäß bei ambulanter Behandlung mit Antidepressiva auf die Therapie an. Zieht man von dieser "hausärztlichen Responsrate» die oben zitierten 20\% ab, so bleibt nach den Regeln der Algebra ein Zweidrittel-Rest übrig, der sich nicht vorrangig mit pharmakodynamischen Effekten erklären lässt. Der dominante Erfolgsanteil in der Praxis resultiert also gar nicht aus der Pharmazie und Pharmakologie der Wirkstoffe, sondern aus den Selbstheilungskräften bzw. deren Stimulation durch das therapeutische Umfeld. Die neuen Empfehlungen im Sinne rationaler Kombinations- bzw. Augmentationsstrategien tragen dieser Erkenntnis verspätetet Rechnung.

Was aber fällt einigen Wettbewerbern aus der parlamentarisch gehegten Phytotherapie dazu ein? Sie berufen eine »Kommission Qualität und Transparenz von Phytopharmaka». Die Ergebnisse von 42 Johanniskraut-Therapiestudien werden selbstzerstörerisch in neun Extrakt-Modifikationen zerpflückt. Während die andere Seite sich mühsam anschickt, von der Wirkstoffdominanz antidepressiver Therapieerfolge abzurücken, gräbt man sich ganz in die Linie von gestern ein und mündet mit einer 53-seitigen statistischen Analyse in den Depressionen auslösenden Satz: "Aufgrund der meist kleinen Zahl von Studien, der unterschiedlichen Patientenpopulationen (leichte, mittelschwere und auch schwere Depressionen, 'major depression' vs. 'nicht major depression'), der unterschiedlichen Vergleichsmöglichkeiten (Placebo, ältere und neuere Antidepressiva) und zum Teil widersprüchlicher Ergebnisse besteht ein erheblicher Interpretationsspielraum, inwieweit der Wirksamkeitsnachweis für einzelne Präparate als vollständig erbracht angesehen werden kann" (3). Die Vertreiber synthetischer Antidepressiva dürften für dieses Eigentor dankbar sein. Teilen und herrschen, mit statistischer Vorarbeit der Gegenpartei! Auf diese Weise wollte der Gesetzgeber den Wettbewerb miteinander konkurrierender Therapierichtungen ganz bestimmt nicht beendet wissen.

Volker Schulz

1 Schwabe U, Paffrath D: Arzneiverordnungs-Report 2000. Berlin, Heidelberg, New York: Springer; 2001: 3.

2 Schwabe U, Paffrath D: Arzneiverordnungs-Report 2002. Berlin, Heidelberg, New York: Springer; 2003: 654.

3 Linde K im Auftrag der Kommission Qualität und Transparenz von Phytopharmaka des Komitees Forschung Naturmedizin e.V.: Johanniskraut bei Depressionen - eine Übersicht der randomisierten Studien bezogen auf einzelne Extrakte. http://www.phytotherapie-komitee.de/ Forschung/kfn_hyperic_281.pdf 